Published in final edited form as:

Ann Inst Stat Math. 2011 February 1; 63(1): 59-80. doi:10.1007/s10463-008-0218-9.

\title{
The local Dirichlet process
}

\author{
Yeonseung Chung and \\ Department of Biostatistics, Harvard School of Public Health, 655 Huntington Ave. Bldg 2, Room \\ 435A, Boston, MA 02115, USA
}

David B. Dunson

Department of Statistical Science, Duke University, 219A Old Chemistry Bldg, Box 90251, Durham, NC 27708, USA, dunson@stat.duke.edu

\begin{abstract}
As a generalization of the Dirichlet process (DP) to allow predictor dependence, we propose a local Dirichlet process (IDP). The IDP provides a prior distribution for a collection of random probability measures indexed by predictors. This is accomplished by assigning stick-breaking weights and atoms to random locations in a predictor space. The probability measure at a given predictor value is then formulated using the weights and atoms located in a neighborhood about that predictor value. This construction results in a marginal DP prior for the random measure at any specific predictor value. Dependence is induced through local sharing of random components. Theoretical properties are considered and a blocked Gibbs sampler is proposed for posterior computation in IDP mixture models. The methods are illustrated using simulated examples and an epidemiologic application.
\end{abstract}

\section{Keywords}

Dependent Dirichlet process; Blocked Gibbs sampler; Mixture model; Non-parametric Bayes; Stick-breaking representation

\section{Introduction}

In recent years, there has been a dramatic increase in applications of non-parametric Bayes methods, motivated largely by the availability of simple and efficient methods for posterior computation in Dirichlet process mixture (DPM) models (Lo 1984; Escobar 1994; Escobar and West 1995). The DPM models incorporate Dirichlet process (DP) priors (Ferguson 1973, 1974) for components in Bayesian hierarchical models, resulting in an extremely flexible class of models. Due to the flexibility and ease in implementation, DPM models are now routinely implemented in a wide variety of applications, ranging from machine learning (Beal et al. 2002; Blei et al. 2004) to genomics (Xing et al. 2004; Kim et al. 2006).

In many settings, it is natural to consider generalizations of the DP and DPM-based models to accommodate dependence. For example, one may be interested in studying changes in a density with predictors. Following Lo (1984), one can use a DPM for Bayes inference on a single density as follows:

\footnotetext{
(C) The Institute of Statistical Mathematics, Tokyo 2009

Correspondence to: Yeonseung Chung.

ychung@hsph.harvard.edu.
} 


$$
f(y)=\int_{\Omega} k(y, u) G(d u),
$$

where $k(y, u)$ is a non-negative valued kernel defined on $(\mathscr{D} \times \Omega, \mathscr{F} \times \mathscr{B})$ such that for each $u \in \Omega, \int_{\mathscr{D}} k(y, u) \mathrm{d} y=1$ and for each $y \in \mathscr{D}, \int_{\Omega} k(y, u) G(d u)<\infty$ with $\mathscr{D}, \Omega$ Borel subsets of Euclidean spaces and $\mathscr{F}, \mathscr{B}$ the corresponding $\sigma$-fields, and $G$ is a finite randomprobabilitymeasure on $(\Omega, \mathscr{B})$ following a DP. A natural extension for modeling of a conditional density $f(y \mid \mathbf{x})$ for $\mathbf{x} \in \mathscr{X}$, with $\mathscr{X}$ a Lebesgue measurable subset of $\Re^{p}$, is as follows:

$$
f(y \mid \mathbf{x})=\int_{\Omega} k(y, u) G_{\mathbf{x}}(d u),
$$

where the mixing measure $G_{\mathbf{x}}$ is now indexed by the predictor value. We are then faced with modeling a collection of random mixing measures denoted as $\mathscr{G}_{\mathscr{X}}=\left\{G_{\mathbf{x}}: \mathbf{x} \in \mathscr{X}\right\}$.

Recent work on defining priors for collections of random probability measures has primarily relied on extending the stick-breaking representation of the DP (Sethuraman 1994). This literature was stimulated by the dependent Dirichlet process (DDP) framework proposed by MacEachern (1999, 2000, 2001), which replaces the atoms in the Sethuraman (1994) representation with stochastic processes. The DDP framework has been adopted to develop ANOVA-type models for random probability measures (De Iorio et al. 2004), for flexible spatial modeling (Gelfand et al. 2004), in time series applications (Caron et al. 2006), and for inferences on stochastic ordering (Dunson and Peddada 2008). The specification of the DDP used in applications incorporates dependence only through the atoms while assuming fixed weights. In other recent work, Griffin and Steel (2006) proposed an order-based DDP ( $\pi \mathrm{DDP})$ which allows varying weights, while Duan et al. (2005) developed a multivariate stick-breaking process for spatial data.

Alternatively, convex combinations of independent DPs can be used for modeling collections of dependent random measures. Müller et al. (2004) proposed this idea to allow dependence across experiments and discrete dynamic settings were considered by Pennell and Dunson (2006) and Dunson (2006). Recently, the idea has been extended to continuous covariate cases by Dunson et al. (2007) and Dunson and Park (2008).

Some desirable properties of a prior for a collection, $\mathscr{G}_{\mathscr{X}}=\left\{G_{\mathbf{x}}: \mathbf{x} \in \mathscr{X}\right\}$, of predictordependent probability measures are: (1) increasing dependence in $G_{\mathbf{x}}$ and $G_{\mathbf{x}^{\prime}}$ with decreasing distance between $\mathbf{x}$ and $\mathbf{x}^{\prime}$; (2) simple and interpretable expressions for the expectation and variance of each $G_{\mathbf{x}}$ as well as the correlation between $G_{\mathbf{x}}$ and $G_{\mathbf{x}}{ }^{\prime} ;$; (3) $G_{\mathbf{x}}$ has a marginal DP prior for all $\mathbf{x} \in \mathscr{X}$; (4) posterior computation can proceed efficiently through a straightforward MCMC algorithm in a broad variety of applications. Although the DDP, $\pi$ DDP and the prior proposed by Duan et al. (2005) achieve (1), $\pi$ DDP and Duan et al. (2005) approaches are not straightforward to implement in general applications. The fixed stick-breaking weights version of the DDP tends to be easy to implement, but has the disadvantage of not allowing locally adaptive mixture weights. The kernel mixture approaches of Dunson et al. (2007) and Dunson and Park (2008) lack the marginal DP property (3). Property (3) is appealing in that there is rich theoretical literature on DPs, showing posterior consistency (Ghosal et al. 1999; Lijoi et al. 2005) and rates of convergence (Ghosal and Van der Vaart 2007).

This article proposes a simple extension of the DP, which provides an alternative to the fixed weights DDP in order to allow local adaptivity, while also achieving properties (1)(4). The prior is constructed by first assigning stick-breaking weights and atoms to random 
locations in a predictor space. Each predictor-dependent random probability measure is formulated using the random weights and atoms located in a neighborhood about that predictor value. Dependence is induced by local sharing of random components. We call this prior the local Dirichlet process (IDP).

Section 2 describes stick-breaking priors (SBP) for collections of predictor-dependent random probability measures. Section 3 introduces the IDP and discusses properties. Computation is described in Sect. 4. Sections 5 and 6 include simulation studies and an epidemiologic application. Section 7 concludes with a discussion. Proofs are included in appendices.

\section{Predictor-dependent stick-breaking priors}

\subsection{Stick-breaking priors}

Ishwaran and James (2001) proposed a general class of SBPs for random probability measures. This class provides a useful starting point in considering extensions to allow predictor dependence.

Definition 1 A random probability measure, $G$, has a SBP if

$$
G=\sum_{h=1}^{N} p_{h} \delta_{\theta_{h}}, \quad 0 \leq p_{h} \leq 1, \quad \sum_{h=1}^{N} p_{h}=1 \quad \text { a.s., } \quad \text { (3) }
$$

where $\delta_{\theta}$ is a discrete measure concentrated at $\theta, p_{h}=V_{h} \prod_{l<h}\left(1-V_{l}\right)$ are random weights with $V_{h} \stackrel{\text { ind }}{\sim} \operatorname{Beta}\left(a_{h}, b_{h}\right)$ independently from $\theta_{h} \stackrel{\text { iid }}{\sim} G_{0}$ with $G_{0}$ a non-atomic base probability measure. For $N=\infty$, the condition $\Sigma_{h=1}^{N} \quad p_{h}=1$ a.s. is satisfied by Lemma 1 in Ishwaran and James (2001). For finite $N$, the condition is satisfied by letting $V_{N}=1$.

There are many processes that fall into this class of SBP. The DP corresponds to the special case in which $N=\infty, a_{h}=1$ and $b_{h}=a$ as established in Sethuraman (1994). The twoparameter Poisson-DP corresponds to the case where $N=\infty, a_{h}=1-a$, and $b_{h}=b+h a$ with $0 \leq a<1$ and $b>-\mathrm{a}$ (Pitman 1995, 1996). Additional special cases are listed in Ishwaran and James (2001).

\subsection{Predictor-dependent stick-breaking priors}

Consider an uncountable collection of predictor-dependent random probability measures, of $\mathscr{G}_{\mathscr{X}}=\left\{G_{\mathbf{x}}: \mathbf{x} \in \mathscr{X}\right\}$. The predictor space $\mathscr{X}$ is Lebesgue measurable subset Euclidian space and the random measures $G_{\mathbf{x}}$ are defined on $(\Omega, \mathscr{B})$ where $\Omega$ is a complete and separable metric space and $\mathscr{B}$ is a corresponding Borel $\sigma$-algebra. Let $\mathscr{P}$ be a probability measure on $(\mathscr{M}, \mathscr{N})$ where $\mathscr{M}$ is the space of uncountable collections of random probability measures $G_{\mathbf{x}}$ and $\mathscr{N}$ is the corresponding Borel $\sigma$-algebra. Then, $\mathscr{G}_{\mathscr{X}} \sim \mathscr{P}$ denotes that $\mathscr{P}$ is a prior on the random collection $\mathscr{G}_{\mathscr{X}}$.

We call $\mathscr{P}$ a predictor-dependent stick-breaking prior $\left(\mathrm{SBP}_{\mathscr{X}}\right)$ if $G_{\mathrm{x}} \in \mathscr{G}_{\mathscr{X}} \sim \mathscr{P}$ can represented as:

$$
\begin{aligned}
G_{\mathbf{x}} & =\sum_{h=1}^{N(\mathbf{x})} p_{h}(\mathbf{x}) \delta_{\theta_{h}(\mathbf{x})} \\
& \text { with } \quad 0 \leq p_{h}(\mathbf{x}) \leq 1 \quad \text { and } \quad \sum_{h=1}^{N(\mathbf{x})} p_{h}(\mathbf{x})=1 \quad \text { a.s., } \forall \mathbf{x} \in \mathscr{X},
\end{aligned}
$$


where the random weights $p_{h}(\mathbf{x})$ have a stick-breaking form, $p_{h}(\mathbf{x})$ and $\theta_{h}(\mathbf{x})$ are predictordependent, and $N(\mathbf{x})$ is also indexed by the predictor value $\mathbf{x}$. Depending on how we form $p_{h}(\mathbf{x}), \theta_{h}(\mathbf{x})$ and $N(\mathbf{x})$, different dependencies among $G_{\mathbf{x}}$ are induced. Several interesting priors, such as the DDP, $\pi \mathrm{DDP}$ and the prior proposed by Duan et al. (2005) fall into the $\mathrm{SBP}_{\mathscr{X}}$ class. In the next section, we propose a new choice of $\mathrm{SBP}_{\mathscr{C}}$ deemed the IDP.

\section{Local Dirichlet process}

\subsection{Formulation}

Formulating the IDP starts with obtaining the following three sequences of mutually independent global random components:

$$
\Gamma=\left\{\Gamma_{h}, h=1, \ldots, \infty\right\}, \mathbf{V}=\left\{V_{h}, h=1, \ldots, \infty\right\}, \Theta=\left\{\theta_{h}, h=1, \ldots, \infty\right\},
$$

where $\Gamma_{h} \stackrel{\text { iid }}{\sim} H$ are locations, $V_{h} \stackrel{\text { iid }}{\sim}$ Beta $(1, \alpha)$ are probability weights, and $\theta_{h} \stackrel{\text { iid }}{\sim} G_{0}$ are atoms. $G_{0}$ is a probability measure on $(\Omega, \mathscr{B})$ on which $G_{\mathbf{x}}$ will be defined and $H$ is a probability measure on $\left(\mathscr{X}^{\prime}, \mathscr{A}\right)$ where $\mathscr{A}$ is a Borel $\sigma$-algebra of subsets of $\mathscr{X}^{\prime}$ and $\mathscr{X}^{\prime}$ is a Lebesgue measurable subset of Euclidian space that may or may not correspond to the predictor space $\mathscr{X}$. For a given predictor space $\mathscr{X}$, we introduce the probability space $\left(\mathscr{X}^{\prime}, \mathscr{A}, H\right)$ such that it satisfies the following regularity condition from which one can deduce $\mathscr{X} \subset \mathscr{X}^{\prime}$ :

Condition 1 For all $\mathbf{x} \in \mathscr{X}$ and $\psi>0, H\left(\eta_{\mathbf{x}}^{\psi}\right)>0$, where $\eta_{\mathbf{x}}^{\psi}=\left\{\mathbf{x}^{\prime}: d\left(x, x^{\prime}\right)<\psi, \mathbf{x}^{\prime} \in \mathscr{X}^{\prime}\right\}$ is defined as a $\psi$-neighborhood around a point $\mathbf{x} \in \mathscr{X}$ with $d: \mathscr{X} \times \mathscr{X}^{\prime} \rightarrow \mathfrak{R}^{+}$being some distance measure.

Next, focusing on a local predictor point $\mathbf{x} \in \mathscr{X}$, we define sets of local random components for $\mathbf{x}$ as:

$$
\Gamma(\mathbf{x})=\left\{\Gamma_{h}, h \in \mathscr{L}_{\mathbf{x}}\right\}, \mathbf{V}(\mathbf{x})=\left\{V_{h}, h \in \mathscr{L}_{\mathbf{x}}\right\}, \Theta(\mathbf{x})=\left\{\theta_{h}, h \in \mathscr{L}_{\mathbf{x}}\right\},
$$

where $\mathscr{L} \mathbf{x}=\left\{h: d\left(\mathbf{x}, \Gamma_{h}\right)<\psi, h=1, \ldots, \infty\right\}$ is a predictor-dependent set indexing the locations belonging to the $\psi$-neighborhood of $\mathbf{x}, \eta_{\mathbf{x}}^{\psi}$, which is defined on $\mathscr{X}^{\prime}$ by $\psi$ and $\mathrm{d}(\cdot, \cdot)$. Hence, the sets $\mathbf{V}(\mathbf{x})$ and $\Theta(\mathbf{x})$ contain the random weights and atoms that are assigned to the locations $\Gamma(\mathbf{x})$ in $\eta_{\mathbf{x}}^{\psi}$. Here, $\psi$ controls the neighborhood size. For simplicity, we treat $\psi$ as fixed throughout the paper, though one can obtain a more flexible class of priors by assuming a hyper prior for $\psi$.

Using the local random components in (6), we consider the following form for $G_{\mathrm{x}}$ :

$$
G_{\mathbf{x}}=\sum_{l=1}^{N(\mathbf{x})} p_{l}(\mathbf{x}) \delta_{\theta_{\pi_{l}(\mathbf{x})}} \quad \text { with } \quad p_{l}(\mathbf{x})=V_{\pi_{l}(\mathbf{x})} \prod_{j<l}\left(1-V_{\pi_{j}(\mathbf{x})}\right),
$$

where $N(\mathbf{x})$ is the cardinality of $\mathscr{L}_{\mathbf{x}}$ and $\pi_{1}(\mathbf{x})$ is the $\ell$ th ordered index in $\mathscr{L}_{\mathbf{x}}$. Then, Condition 1 ensures that the following lemma holds (refer to the Proof of Lemma 1 in the Appendix).

Lemma 1 For all $\mathbf{x} \in \mathscr{X}, N(\mathbf{x})=\infty$ and $\sum_{l=1}^{N(\mathbf{x})} p_{l}(\mathbf{x})=1$ almost surely.

By Lemma 1, it is apparent that $G_{\mathbf{X}}$ formed as in (7) is a well-defined stick-breaking random probability measure for $\mathbf{x}$. It is also straightforward that we can define $G_{X}$ for all $\mathbf{x} \in \mathscr{X}$ by (6) and (7) using the global components in (5). Therefore, given $a, G_{0}, H, \psi$ ) with a choice of $d(\cdot, \cdot)$, the steps from (5) to (7) defines a new choice of predictor-dependent $\operatorname{SBP}\left(\operatorname{SBP}_{\mathscr{X}}\right)$ 
for $\mathscr{G}_{\mathscr{X}}$, deemed the IDP. We use the shorthand notation $\mathscr{G}_{\mathscr{X}}=\left\{G_{\mathbf{x}}: \mathbf{x} \in \mathscr{X}\right\} \sim \operatorname{IDP}\left(a, G_{0}, H\right.$, $\psi)$ to denote that $\mathscr{G}_{\mathscr{X}}$ is assigned a IDP with hyperparameters $a, G_{0}, H, \psi$.

Figure 1 illustrates the IDP formulation graphically for a case where $\mathscr{X}=[0,1]^{2}$ and $\mathscr{G}_{\mathscr{X}} \operatorname{IDP}\left(\alpha, G_{0}, H, \psi\right)$ with $H=\mathrm{Uniform}\left([0,1]^{2}\right)$ leading to $\mathscr{X}=\mathscr{X}^{\prime}$ and $\psi=0.2$. For a simple illustration, we consider Euclidian distance for $d(\cdot, \cdot)$ for bivariate predictors. Random locations in $[0,1]^{2}$ are generated from a uniform distribution, with the first 100 locations plotted as ' $*$ ' in Fig. 1. The random pair of weight and atom $\left(V_{\mathrm{h}}, \theta_{h}\right)$ is placed at location $\Gamma_{h}$, with the first ten pairs labeled in Fig. 1. For a predictor value $\mathbf{x}=(0.5,0.3)^{\prime}$, the red dashed circle indicates the neighborhood of $\mathbf{x}, \eta_{\mathbf{x}}^{\psi}$. Then, $G_{\mathrm{x}}$ at $\mathbf{x}=(0.5,0.3)^{\prime}$ is constructed using the weights and atoms within the dashed circle in the order of the index to formulate the stick-breaking representation. For all other $\mathbf{x} \in \mathscr{X}, G_{\mathrm{x}}$ are formed following the same steps.

From Fig. 1, it is apparent that the dependence between $G_{\mathbf{x}}$ and $G_{\mathbf{x}^{\prime}}$ increases as the distance between $\mathbf{x}$ and $\mathbf{x}^{\prime}$ decreases. For closer $\mathbf{x}$ and $\mathbf{x}^{\prime}$, their neighborhoods overlap more so that similar components are used for constructing $G_{\mathrm{x}}$ and $G_{\mathrm{x}^{\prime}}$, while if $\mathbf{x}$ and $\mathbf{x}^{\prime}$ are far apart, there will be at most a small area of intersection so that few to none of the random components are shared. In the non-overlapping case, $G_{\mathbf{x}}$ and $G_{\mathbf{x}}^{\prime}$ are assigned independent DP priors, as is clear from Theorem 1 and the subsequent development.

Theorem 1 If $\mathscr{G}_{\mathscr{X}} \mathcal{I} D P\left(\alpha, G_{0}, H, \psi\right)$, for any $\mathbf{x} \in \mathscr{X}, G_{\mathbf{x}} \sim D P\left(a G_{0}\right)$.

The marginal DP property shown in Theorem 1 is appealing in allowing one to rely directly on the rich literature on properties of the DP to obtain insight into the prior for the random probability measure at any particular predictor value. However, unlike the DP, the IDP allows the probability measure to vary with predictors, while borrowing information across local regions of the predictor space. This is accomplished through incorporating shared random components. Due to the sharing and to the almost sure discreteness property of each $G_{\mathbf{x}}$, the IDP will induce local clustering of subjects according to their predictor values. Theorem 2 illustrates this local clustering property more clearly.

Theorem 2 Suppose $\mathscr{G}_{\mathscr{X}} T D P\left(\alpha, G_{0}, H, \psi\right)$ and $\phi_{i} \mid G_{\mathbf{x}_{i}}$ ind $G_{x_{i}}$, for $i=1, \ldots, n$, with $\mathbf{x}_{i}$ denoting the predictor value for subject $i$. Then,

$$
\kappa_{\mathbf{x}_{i}, \quad \mathbf{x}_{j}}=\operatorname{Pr}\left(\phi_{i}=\phi_{j} \mid \mathbf{x}_{i}, \mathbf{x}_{j}, \alpha, \psi\right)=\frac{2 P_{\mathbf{x}_{i}, \mathbf{x}_{j}}}{\left(1+P_{\mathbf{x}_{i}, \mathbf{x}_{j}}\right) \alpha+2}, \text { for } \quad \text { any } \quad \mathbf{x}_{i}, \mathbf{x}_{j} \in \mathscr{X},
$$

$$
\begin{aligned}
& { }_{\text {where }} P_{\mathbf{x}_{i}, \mathbf{x}_{j}}=\frac{H\left(\eta_{\mathbf{x}_{i}}^{\psi} \cap \eta_{\mathbf{x}_{j}}^{\psi}\right)}{H\left(\eta_{\mathbf{x}_{i}}^{\psi} \cup \eta_{\mathbf{x}_{j}}^{\psi}\right)} \text { is the conditional probability of } \Gamma_{h} \text { falling within the intersection } \\
& \text { region } \eta_{\mathbf{x}_{i}}^{\psi} \cap \eta_{\mathbf{x}_{j}}^{\psi} \text { given } \Gamma_{h} \in \eta_{\mathbf{x}_{i}}^{\psi} \cup \eta_{\mathbf{x}_{j} .}^{\psi}
\end{aligned}
$$

The clustering probability $\boldsymbol{\kappa}_{\mathbf{x}_{i}, \mathbf{x}_{j}}$ increases from 0 when $\eta_{\mathbf{x}_{i}}^{\psi} \cap \eta_{\mathbf{x}_{j}}^{\psi}=\oslash$ to $1 /(a+1)$ when $\mathbf{x}_{i}=\mathbf{x}_{j}$ which is the case of $P_{\mathbf{x}_{i}, \mathbf{x}_{j}}=1$. This implies that, for fixed $a$, the clustering probability under $\mathscr{G}_{\mathscr{X}} \operatorname{TDP}\left(\alpha, G_{0}, H, \psi\right)$ is bounded above by the clustering probability under the global DP, which takes $G_{\mathbf{X}} \equiv G \mathrm{DP}\left(\alpha G_{0}\right)$, leading to $\operatorname{Pr}\left(\varphi_{i}=\varphi_{j} \mid a\right)=1 /(a+1)$. Also, note that small values of the precision parameter $a$ will induce $V_{h}$ values that are close to one. This in turn causes a small number of atoms in each neighborhood to dominate, inducing few local 
clusters. However, when $\psi$ is small and hence neighborhood sizes are small, there will still be many clusters across $\mathscr{X}$.

It is interesting to consider relationships between the IDP and other priors proposed in the literature in limiting special cases. First, note that the IDP converges to the DP as $\psi \rightarrow \infty$, so that all the neighborhoods around each of the predictor values encompass the entire predictor space. Also, the $\operatorname{lDP}\left(a, G_{0}, H, \psi\right)$ corresponds to a limiting case of the kernel stick-breaking process (KSBP) (Dunson and Park 2008), in which the kernel is defined as $K(\mathbf{x}, \Gamma)=1(d(\mathbf{x}, \Gamma)<\psi)$ and the DP placed at each location have precision parameters $\rightarrow 0$.

\subsection{Moments and correlation}

From Theorem 1 and properties of the DP, $\mathscr{G}_{\mathscr{X}} \operatorname{TDP}\left(\alpha, G_{0}, H, \psi\right)$ implies, for any $\mathbf{x} \in \mathscr{X}$,

$$
E\left\{G_{\mathbf{x}}(B)\right\}=G_{0}(B) \quad \text { and } \quad \operatorname{Var}\left\{G_{\mathbf{x}}(B)\right\}=\frac{G_{0}(B)\left(1-G_{0}(B)\right)}{1+\alpha}, \quad \forall B \in \mathscr{B}
$$

Next, let us consider the correlation between $G_{\mathbf{x}_{1}}$ and $G_{\mathbf{x}_{2}}$, for any $\mathbf{x}_{1}, \mathbf{x}_{2} \in \mathscr{X}$. First, we show the correlation conditionally on the locations $\Gamma$ but marginalizing out the weights $\mathbf{V}$ and atoms $\Theta$. As discussed in Sect. 3.1, if $\Gamma$ is given, the IDP can be regarded as a special case of the $\pi$ DDP. Hence, following Theorem 1 in Griffin and Steel (2006), for any $\mathbf{x}_{1}$, $\mathbf{x}_{2} \in \mathscr{X}$,

$$
\begin{aligned}
& \rho_{\mathbf{x}_{1}, \mathbf{x}_{2}}(\Gamma)=\operatorname{Corr}\left\{G_{\mathbf{x}_{1}}(B), G_{\mathbf{x}_{2}}(B) \mid \Gamma\right\} \\
& =\frac{2}{\alpha+2} \sum_{h \in \mathscr{L}_{\mathbf{x}_{1}} \cap \mathscr{L}_{\mathbf{x}_{2}}}\left(\frac{\alpha}{\alpha+2}\right)^{\# \mathscr{S}_{h}}\left(\frac{\alpha}{\alpha+1}\right)^{\# \mathscr{S}_{h}^{\prime}}, \quad \forall \quad B \in \mathscr{B},
\end{aligned}
$$

where \#S is the cardinality of the set $\mathscr{S}, \mathscr{S}_{h}=\mathscr{A}_{1 h} \cap \mathscr{A}_{2 h}, \mathscr{S}_{h}^{\prime}=\mathscr{A}_{1 h} \cup \mathscr{A}_{2 h}-\mathscr{S}_{h}$, and $\mathscr{A}_{k h}=\left\{\pi_{j}\left(\mathbf{x}_{k}\right): j<l, \pi_{l}\left(\mathbf{x}_{k}\right)=h\right\}$ for $h \in \mathscr{L}_{\mathbf{x}_{1}} \cap \mathscr{L}_{\mathbf{x}_{2}}$. In other words, \# $S_{h}$ is the number of indices on the locations $\Gamma$ that are below $h$ and are shared in the neighborhoods of $\mathbf{x}_{1}$ and $\mathbf{x}_{2}$, while $\# \mathscr{S}_{h}^{\prime}$ is the number of indices that are below $\mathrm{h}$ and belong to the neighborhoods of either $\mathbf{x}_{1}$ or $\mathbf{x}_{2}$ but not both. For a given $h$, reducing $\# S_{h}$ by one induces adding two elements to $\mathscr{S}_{h}^{\prime}$, thus reducing the correlation, as expected. From expression (9), it is clear that the neighborhoods around $\mathbf{x}_{1}$ and $\mathbf{x}_{2}$ are increasingly overlapping and the correlation between $G_{\mathbf{x}_{1}}$ and $G_{\mathbf{x}_{2}}$ increases as $\mathbf{x}_{1} \rightarrow \mathbf{x}_{2}$. Expression (9) is particularly useful in being free of dependence on $\mathrm{B}$.

Marginalizing the correlation in (9) over the prior for the random locations $\Gamma$ is equivalent to marginalizing out the $\# S_{h}$ and $\# \mathscr{S}_{h}^{\prime}$ for $h \in \mathscr{L}_{\mathbf{x}_{1}} \cap \mathscr{L}_{\mathbf{x}_{2}}$. In considering the correlation between $G_{\mathrm{x}_{1}}$ and $G_{\mathrm{x}_{2}}$, we can ignore the $\Gamma_{h}$ for $h \in\{1, \ldots, \infty\} \backslash \mathscr{L}_{\mathrm{x}_{1}} \cup \mathscr{L}_{\mathrm{x}_{2}}$ and focus on the $\Gamma_{h}$ only for $h \in \mathscr{L}_{\mathbf{x}_{1}} \cup \mathscr{L}_{\mathbf{x}_{2}}$. Let $\gamma_{j}$ be the $j$ th ordered component of $\mathscr{L}_{\mathbf{x}_{1}} \cup \mathscr{L}_{\mathbf{x}_{2}}$. For example, if $\mathscr{L}_{\mathbf{x}_{1}} \cup \mathscr{L}_{\mathbf{x}_{2}}=\{1,3,5,6,7,8, \ldots\}, \gamma_{1}=1, \gamma_{2}=3, \gamma_{3}=5, \gamma_{4}=6, \ldots$ Let $Z_{\gamma_{j}}=1\left(\gamma_{j} \in \mathscr{L}_{\mathbf{x}_{1}} \cap \mathscr{L}_{\mathbf{x}_{2}}\right)$ be an indicator for whether $\Gamma_{\gamma j}$ are shared by the neighborhoods of $\mathbf{x}_{1}$ and $\mathbf{x}_{2}$ or not. Then, the formula in (9) can be reexpressed with respect to $Z_{\gamma j}$ as follows:

$$
\begin{aligned}
\rho_{\mathbf{x}_{1}, \mathbf{x}_{2}}(\Gamma) & =\operatorname{Corr}\left\{G_{\mathbf{x}_{1}}(B), G_{\mathbf{x}_{2}}(B) \mid \Gamma\right\} \\
& =\frac{2}{\alpha+2} \sum_{j=1}^{\infty} Z_{\gamma_{j}}\left(\frac{\alpha}{\alpha+2}\right)^{\Sigma_{k=1}^{j-1} Z_{\gamma_{k}}}\left(\frac{\alpha}{\alpha+1}\right)^{j-1-\Sigma_{k=1}^{j-1} Z_{\gamma_{k}}} .
\end{aligned}
$$


Note that it is straightforward to show that $Z_{\gamma_{j}} \stackrel{\text { iid }}{\sim} \operatorname{Bernoulli}\left(P_{\mathbf{x}_{1}, \mathbf{x}_{2}}\right) \operatorname{Bernoulli}\left(P_{\mathbf{x}_{1}, \mathbf{x}_{2}}\right)$, for $j=$ $1, \ldots, \infty$, with $P_{\mathbf{x}_{1}, \mathbf{x}_{2}}=\frac{H\left(\eta_{\mathbf{x}_{1}}^{\psi} \cap \eta_{\mathbf{x}_{2}}^{\psi}\right)}{H\left(\eta_{\mathbf{x}_{1}}^{\psi} \cup \eta_{\mathbf{x}_{2}}^{\psi}\right)}$ the conditional probability of $\Gamma_{h}$ falling within the intersection region $\eta_{\mathbf{x}_{1}}^{\psi} \cap \eta_{\mathbf{x}_{2}}^{\psi}$ given $\Gamma_{h} \in \eta_{\mathbf{x}_{1}}^{\psi} \cup \eta_{\mathbf{x}_{2}}^{\psi}$. Finally, marginalizing out $\left\{Z_{\gamma_{j}}\right\}_{j=1}^{\infty}$ results in the following Theorem.

Theorem 3 If $\mathscr{G}_{\mathscr{X}} \mathcal{I D P}\left(\alpha, G_{0}, H, \psi\right)$, for any $\mathbf{x}_{1}, \mathbf{x}_{2} \in \mathscr{X}$,

$$
\rho_{\mathbf{x}_{1}, \mathbf{x}_{2}}=\operatorname{Corr}\left\{G_{\mathbf{x}_{1}}(B), G_{\mathbf{x}_{2}}(B)\right\}=\frac{2 P_{\mathbf{x}_{1}, \mathbf{x}_{2}}(\alpha+1)}{\left(1+P_{\mathbf{x}_{1}, \mathbf{x}_{2}}\right) \alpha+2}, \quad \forall \quad B \in \mathscr{B} .
$$

The correlation is expressed only in terms of $P_{\mathbf{x}_{1}, \mathbf{x}_{2}}$ and $a$. Regardless of $a$, the correlation is 1 if $\mathbf{x}_{1}=\mathbf{x}_{2}$ which implies the neighborhoods around $\mathbf{x}_{1}, \mathbf{x}_{2}$ are identical and $P_{\mathbf{x}_{1}, \mathbf{x}_{2}}=1$. Also, the correlation is 0 when the neighborhoods are non-overlapping with $P_{\mathbf{x}_{1}, \mathbf{x}_{2}}=0$. In addition, $P_{\mathbf{x}_{1}, \mathbf{x}_{2}} \leq \rho_{\mathbf{x}_{1}, \mathbf{x}_{2}} \leq 1$ and $\rho_{\mathbf{x}_{1}, \mathbf{x}_{2}}$ increases as $a$ increases for fixed $P_{\mathbf{x}_{1}, \mathbf{x}_{2}}$. When $a \rightarrow 0$, the correlation converges to $P_{\mathbf{x}_{1}, \mathbf{x}_{2}}$. Meanwhile, when $a \rightarrow \infty$, the correlation converges to $\frac{2 P_{\mathbf{x}_{1}, \mathbf{x}_{2}}}{1+P_{\mathbf{x}_{1}, \mathbf{x}_{2}}}$.

Note that $P_{\mathbf{x}_{1}, \mathbf{x}_{2}}$ depends on $H, \psi$, and the locations $\mathbf{x}_{1}$ and $\mathbf{x}_{2}$ given a choice of $d(\cdot, \cdot)$. When $\mathscr{X}^{\prime}$ for $H$ is chosen to satisfy Condition 2, some appealing properties result.

Condition 2 For all $\mathbf{x} \in \mathscr{X}$ with $\mathscr{X}$ being $p$-dimensional, $\left\{\mathbf{x}^{*} ; d\left(\mathbf{x}^{*}, \mathbf{x}\right)<\psi, \mathbf{x}^{*} \in \mathfrak{R}^{p}\right\} \subset \mathscr{X}^{\prime}$.

From Condition 2, one can deduce that $\mathscr{X}^{\prime}$ contains all the points in $\mathfrak{R}^{p}$ within the distance of $\psi$ from $\mathbf{x}$ for any $\mathbf{x} \in \mathscr{X}$. Under Condition 2, with $H$ chosen to be a uniform probability measure on a bounded space $\mathscr{X}^{\prime}, P_{\mathbf{x}_{1}, \mathbf{x}_{2}}$ depends only on $\psi$ and $d\left(\mathbf{x}_{1}, \mathbf{x}_{2}\right)$ which is the distance between $\mathbf{x}_{1}$ and $\mathbf{x}_{2}$, but not on the exact locations of $\mathbf{x}_{1}$ and $\mathbf{x}_{2}$ in $\mathscr{X}$. Hence, upon examination of Theorem 3, it is apparent that Condition 2 implies an isotropic correlation structure, which is an appealing default in the absence of prior knowledge of changes in the correlation structure according to the locations in $\mathscr{X}$. Figure 2 shows how the correlation $\rho_{\mathbf{x}_{1}, \mathbf{x}_{2}}$ changes as a function of $d\left(\mathbf{x}_{1}, \mathbf{x}_{2}\right)$ in the case where $\mathbf{x} \in \mathscr{X}=[0,1]$ and $H$ is Uniform $([-\psi, 1+\psi])$ so that $\mathscr{X}^{\prime}=[-\psi, 1+\psi]$ and Condition 2 holds for different $\psi$ with $d(\cdot$, .) corresponding to the Euclidian distance. The $\rho_{\mathbf{x}_{1}, \mathbf{x}_{2}}$ decays from 1 to 0 as $d\left(\mathbf{x}_{1}, \mathbf{x}_{2}\right)$ increases and the decay is faster for smaller $\psi$. As $\psi \rightarrow \infty$, the decay line gets closer to a horizontal line at $\rho_{\mathrm{x}_{1}, \mathrm{x}_{2}}=1$, which is the case of 1DP=DP. Also, for a given $\psi$ and $d\left(\mathbf{x}_{1}, \mathbf{x}_{2}\right)$, the $\rho_{\mathrm{x}_{1}, \mathrm{x}_{2}}$ is higher as $a \rightarrow \infty$. Although the choice of $d(\cdot, \cdot)$ being Euclidian makes the curves in Fig. 2 close to linear, the curvature can easily be changed by choosing a different distance measure $d(\cdot, \cdot)$.

\subsection{Truncation approximation}

Finite approximations to infinite SBPs form the basis for commonly used computational algorithms (Ishwaran and James 2001). In this subsection, we discuss a finite dimensional approximation to the IDP.

Since the IDP has the marginal DP property, let us recall the finite dimensional DP. Ishwaran and James (2001) defines an $N$-truncation of the $\mathrm{DP}\left(\mathrm{DP}^{N}\right)$ by discarding the $N+$ $1, N+2, \ldots, \infty$ terms and replacing $p_{N}$ with $1-\sum_{h=1}^{N-1} p_{h}$ in the DP stick-breaking form in 
(3). They show that the $\mathrm{DP}^{N}$ approximates the DP well in terms of the total variation (tv) norm of the marginal densities of the data obtained from the corresponding DPM models. According to their Theorem 2,

$$
\left\|\mu_{N}-\mu_{\infty}\right\| \leq 4\left[1-E\left\{\left(\sum_{h=1}^{N-1} p_{h}\right)^{n}\right\}\right] \approx 4 n \times \exp \{-(N-1) / \alpha\},
$$

where $\|\cdot\|$ is tv norm, $\mu_{N}$ and $\mu_{\infty}$ are the marginal probability measures for the data from the $\mathrm{DPM}^{N}$ and DPM models, and $n$ is the sample size. Note that the sample size has a modest effect on the bound for a reasonably large value of $N$ and the bound decreases exponentially with $N$ increasing, implying that even for a fairly large sample size, the $\mathrm{DPM}^{N}$ approximates the DP well with moderate $N$.

Following a similar route, let us define an $N$-truncation of the $\operatorname{lDP}\left(\operatorname{lDP}^{N}\right)$ as follows:

Definition 2 For a finite $N$, let $\Gamma^{N}=\left\{\Gamma_{h}, h=1, \ldots, N\right\}, \mathbf{V}^{N}=\left\{V_{\mathrm{h}}, h=1, \ldots, N\right\}$, and $\Theta^{N}=$ $\left\{\theta_{h}, h=1, \ldots, N\right\}$ be the sets of global random locations, weights, and atoms, respectively. Distributional assumptions for $\Gamma_{h}, V_{\mathrm{h}}$, and $\theta_{h}$ are the same as in (5) and the corresponding local sets are defined as in (6). Then, $\mathscr{G}_{\mathscr{X}} \mathcal{T} P^{N}\left(\alpha, G_{0}, H, \psi\right)$ if

$$
\begin{aligned}
G_{\mathbf{x}} & =\sum_{l=1}^{N(\mathbf{x})-1} p_{l}(\mathbf{x}) \delta_{\theta_{\pi_{l}(\mathbf{x})}}+\left(1-\sum_{l=1}^{N(\mathbf{x})-1} p_{l}(\mathbf{x})\right) \delta_{\theta_{\pi_{N(\mathbf{x})}(\mathbf{x})}} \\
& \text { with } \quad p_{l}(\mathbf{x})=V_{\pi_{l}(\mathbf{x})} \prod_{j<1}\left(1-V_{\left.\pi_{j}(\mathbf{x})\right)} \text { for } l=1, \ldots, N(\mathbf{x})-1 .\right.
\end{aligned}
$$

The $G_{\mathbf{x}}$ in Definition 3 has a similar form to $G=\sum_{h=1}^{N} p_{h} \delta_{\theta_{h}}$ obtained from the $\mathrm{DP}^{N}$ except that $N$ in $G$ is replaced by $N(\mathbf{x})$ in $G_{\mathbf{x}}$ and $\mathrm{N}$ in $\mathrm{DP}^{N}$ is fixed while $N(\mathbf{x})$ in $\operatorname{lDP}^{N}$ is random. Focusing on a particular predictor value $\mathbf{x}$, it is easy to show that $N(\mathbf{x}) \sim \operatorname{Binomial}\left(N, P_{\mathrm{X}}\right)$, where $N$ is the total number of global locations in $\operatorname{IDP}^{N}$ and $P_{\mathbf{x}}=H\left(\eta_{\mathbf{x}}^{\psi}\right)$ is the probability that a location belongs to the neighborhood around $\mathbf{x}, \eta_{\mathbf{x}}^{\psi}$. Then, marginalizing out $N(\mathbf{x})$ in the bound on the tv distance between the marginal densities of an observation obtained at a particular predictor value $\mathbf{x}$ from the IDPM and $1 \mathrm{DPM}^{N}$ models results in Theorem 4 .

Theorem 4 Define a model (2) with $\mathscr{G}_{\mathscr{X}} \mathcal{I D P}\left(\alpha, G_{0}, H, \psi\right)$ as local Dirichlet process mixture (IDPM) model). IDPM ${ }^{N}$ corresponds to (2) with $\mathscr{G}_{\mathscr{X}}{ }{ }^{2} P^{N}\left(\alpha, G_{0}, H, \psi\right)$. Suppose an observation is obtained from IDPMN and IDPM models at $\mathbf{x}$. Then,

$$
\left\|\mu_{N}(\mathbf{x})-\mu_{\infty}(\mathbf{x})\right\| \leq 4\left(\frac{\alpha+1}{\alpha}\right)\left\{1-\left(\frac{1}{\alpha+1}\right) P_{\mathbf{x}}\right\}^{N}
$$

where $\mu_{N}(\mathbf{x})$ and $\mu_{\infty}(\mathbf{x})$ are the marginal probability measures for the observation. Notice that the bound decreases exponentially with $\mathrm{N}$ increasing, suggesting that we can obtain a good approximation to the IDP using a moderate $\mathrm{N}$, as long as $a$ is small and the neighborhood size is not too small. In particular, we require a large $\mathrm{N}$ for a given level of accuracy as $\psi \rightarrow 0$, since $P_{\mathbf{x}}$ decreases as the size of $\eta_{\mathbf{x}}^{\psi}$ decreases. 


\section{Posterior computation}

We develop an MCMC algorithm based on the blocked Gibbs sampler (Ishwaran and James 2001) for an $\mathrm{IDPM}^{N}$ model. For simplicity in exposition, we describe a Gibbs sampling algorithm for a particular hierarchical model, though the approach can be easily adapted for computation in a broad variety of other settings. We let

$$
\begin{aligned}
f\left(y_{i} \mid \mathbf{x}_{i}, \tau\right) & =\int f\left(y_{i} \mid \mathbf{x}_{i}, \beta_{i}, \tau\right) \mathrm{d} G_{\mathbf{x}_{i}}\left(\beta_{i}\right) \text { for } \quad i=1, \ldots, n \\
\mathscr{G}_{\mathscr{X}} & \operatorname{IDP}^{N}\left(\alpha, G_{0}, H, \psi\right),
\end{aligned}
$$

where $f\left(y_{i} \mid \mathbf{x}_{i}, \boldsymbol{\beta}_{i}, \boldsymbol{\tau}\right)=N\left(y_{i}, \mathbf{x}_{i}^{\prime}, \boldsymbol{\beta}_{i}, \tau^{-1}\right) \boldsymbol{\beta}_{i}=\left(\beta_{i 1}, \ldots, \beta_{i p}\right)^{\prime}$. For simplicity, we consider a univariate predictor case where $p=2$ and $\mathbf{x}_{i}^{\prime}=\left(1, x_{i}\right)$ with $d(\cdot, \cdot)$ Euclidian distance but the generalization to multiple predictors or to using different distance metric is straightforward. $G_{0}$ is assumed to be $N_{p}\left(\mu_{\beta}, \Sigma_{\beta}\right), H$ is assumed to be Uniform $\left(a_{\Gamma}, b_{\Gamma}\right)$ and additional conjugate priors are assigned for $\tau, a, \mu_{\beta}$ and $\Sigma_{\beta}$.

Let $K_{i}$ be an indicator variable denoting that $K_{i}=h$ implies $i$ th subject is assigned to the $h$ th mixture component. Then, the hierarchical structure of the model (12) with respect to the random variables is recast as follows.

$$
\begin{aligned}
\left(y_{i} \mid \mathbf{x}_{i}, \beta^{*}, \tau, \mathbf{K}\right. & \mathcal{N}\left(\mathbf{x}_{i}^{\prime} \beta_{K_{i}}^{*}, \tau^{-1}\right), \quad i=1, \ldots, n \\
\left(K_{i} \mid \mathbf{V}, \Gamma\right) & \stackrel{N}{N\left(\mathbf{x}_{i}\right)} p_{l=1}\left(\mathbf{x}_{i}\right) \delta_{\pi_{l}\left(\mathbf{x}_{i}\right)}(\cdot), \quad i=1, \ldots, n \\
\left(V_{h} \mid \alpha\right. & \operatorname{Beta}(1, \alpha), \quad h=1, \ldots, N \\
\left(\Gamma_{h}\right) & \operatorname{Uniform}\left(a_{\Gamma}, b_{\Gamma}\right) \quad h=1, \ldots, N \\
\left(\beta_{h}^{*} \mid \mu_{\beta}, \Sigma_{\beta}\right) & N_{p}\left(\mu_{\beta}, \Sigma_{\beta}\right), \quad h=1, \ldots, N \\
\mu_{\beta} & N_{p}\left(\mu_{0}, \Sigma_{\mu}\right) \\
\Sigma_{\beta}^{-1} & \text { Wishart }\left(\left\{v_{0} \Sigma_{0}\right\}^{-1}, v_{0}\right) \\
\tau & \operatorname{Gamma}\left(v_{1}, v_{2}\right) \\
\alpha & \operatorname{Gamma}\left(\eta_{1}, \eta_{2}\right),
\end{aligned}
$$

where $\beta^{*}=\left\{\beta_{h}^{*}, h=1, \ldots, N\right\}, \mathbf{K}=\left\{K_{i}, i=1 \ldots, n\right\}, \mathbf{V}=\left\{V_{h}, h=1, \ldots, N\right\}$, and $\Gamma=\left\{\Gamma_{h}, h=\right.$ $1, \ldots, N\}$. The full conditionals for each of the random components are based on the following joint distribution.

$$
\left(\mathbf{y}, \mathbf{K}, \mathbf{V}, \Gamma, \beta^{*}, \mu_{\beta}, \Sigma_{\beta}, \tau, \alpha\right) \propto\left(\mathbf{y} \mid \beta^{*}, \tau, \mathbf{K}\right)(\mathbf{K} \mid \mathbf{V}, \Gamma)(\mathbf{V} \mid \alpha)(\Gamma)\left(\beta^{*} \mid \mu_{\beta}, \Sigma_{\beta}\right)\left(\mu_{\beta}\right)\left(\Sigma_{\beta}\right)(\tau)(\alpha)
$$

Then, the Gibbs sampler proceeds by sampling from the following conditional posterior distributions:

a. Conditional for $K_{i}, i=1, \ldots, n$

$$
\begin{aligned}
\left(K_{i} \mid \mathbf{y}, \mathbf{V}, \Gamma, \beta^{*}, \tau\right. & \stackrel{\sim}{\sim} \sum_{l=1}^{N\left(\mathbf{x}_{i}\right)} p_{l}^{\prime}\left(\mathbf{x}_{i}\right) \delta_{\pi_{l}\left(\mathbf{x}_{i}\right)}\left(K_{i}\right) \\
p_{l}^{\prime}\left(\mathbf{x}_{i}\right) & =\frac{N\left(y_{i}, \mathbf{x}_{i}^{\prime} \beta_{\pi_{l}}^{*}\left(\mathbf{x}_{i}\right) \tau^{-1}\right) p_{l}\left(\mathbf{x}_{i}\right)}{\sum_{l=1}^{N\left(\sum_{i}\right)} N\left(y_{i} ; \mathbf{x}_{i}^{\prime} \beta_{\pi_{l}}^{*}\left(\mathbf{x}_{i}, \tau^{-1}\right) p_{l}\left(\mathbf{x}_{i}\right)\right.} \\
p_{l}\left(\mathbf{x}_{i}\right) & =V_{\pi_{l}\left(\mathbf{x}_{i}\right)} \prod_{j<l}\left(1-V_{\pi_{j}\left(\mathbf{x}_{i}\right)}\right) \quad \text { for } \quad l<N\left(\mathbf{x}_{i}\right) \\
p_{l}\left(\mathbf{x}_{i}\right) & =\prod_{j<1}\left(1-V_{\pi_{j}\left(\mathbf{x}_{i}\right)}\right) \quad \text { for } \quad l=N\left(\mathbf{x}_{i}\right)
\end{aligned}
$$


b. Conditional for $V_{h}, h=1, \ldots, N$

$$
\left(V_{h} \mid \mathbf{K}, \Gamma, \alpha\right) \operatorname{Beta}\left(1+\sum_{i=1}^{n} 1\left(K_{i}=h \quad \text { and } \quad K_{i} \neq \pi_{N\left(x_{i}\right)}\left(\mathbf{x}_{i}\right)\right), \alpha+\sum_{i=1}^{n} 1\left(K_{i}>h\right)\right)
$$

c. Conditional for $\Gamma_{h}, h=1, \ldots, N$

$$
\left(\Gamma_{h} \mid \mathbf{K}, \mathbf{V}\right) \text { Uniform }\left(\max \left[\max _{i=K_{i}=h}\left(x_{i}-\psi\right), a_{\Gamma}\right], \min \left[\min _{i=K_{i}=h}\left(x_{i}+\psi\right), a_{\Gamma}\right]\right)
$$

d. Conditional for $\beta_{h}^{*}, h=1, \ldots, N$

$$
\begin{aligned}
\left(\beta_{h}^{*} \mid \mathbf{y}, \mathbf{K}, \mu_{\beta}, \Sigma_{\beta}, \tau\right) & N_{p}\left(\widehat{\mu}_{\beta h}, \widehat{\Sigma}_{\beta h}\right) \\
\widehat{\mu}_{\beta h} & =\widehat{\Sigma}_{\beta h}\left[\Sigma_{\beta}^{-1} \mu_{\beta}+\tau \mathbf{X}_{i h} \mathbf{y}_{i h}\right] \\
\widehat{\Sigma}_{\beta h} & =\left[\Sigma_{\beta}{ }^{-1}+\tau \mathbf{X}_{i h} \mathbf{X}_{i h}^{\prime}\right]^{-1}
\end{aligned}
$$

where $\mathbf{y}_{i h}$ is $n_{h} \times 1$ response vector and $\mathbf{X}_{i h}^{\prime}$ is $n_{h} \times p$ design matrix for the subjects with $K_{i}=h$ and $n_{h}$ is the number of those subjects.

e. Conditional for $\boldsymbol{\mu}_{\beta}$

$$
\begin{gathered}
\left(\mu_{\beta} \mid \beta^{*}, \Sigma_{\beta}\right) N_{p}\left(\widehat{\mu}_{0}, \widehat{\Sigma}_{\mu}\right) \\
\widehat{\mu}_{0}=\widehat{\Sigma}_{\mu}\left[\Sigma_{\mu}^{-1} \mu_{0}+\Sigma_{\beta}^{-1} \sum_{h=1}^{N} \beta_{h}^{*}\right] \\
\widehat{\Sigma}_{\mu}=\left[\Sigma_{\mu}^{-1}+N \Sigma_{\beta}^{-1}\right]^{-1}
\end{gathered}
$$

f. Conditional for $\sum_{\beta}^{-1}$

$$
\left(\Sigma_{\beta}^{-1} \mid \beta^{*}, \mu_{\beta}\right) \text { Wishart }\left(\left[\sum_{h=1}^{N}\left(\beta_{h}^{*}-\mu_{\beta}\right)\left(\beta_{h}^{*}-\mu_{\beta}\right)^{\prime}+v_{0} \Sigma_{0}\right]^{-1}, N+v_{0}\right)
$$

g. Conditional for $\tau$

$$
\left(\tau \mid \mathbf{y}, \beta^{*}, \mathbf{K}\right)^{\text {Gamma }}\left(v_{1}+\frac{n}{2}, v_{2}+\frac{1}{2} \sum_{i=1}^{n}\left(y_{i}-\mathbf{x}_{i}^{\prime} \beta_{K_{i}}^{*}\right)^{2}\right)
$$

h. Conditional for $a$

$$
(\alpha \mid \mathbf{V}) \text { Gamma }\left(\eta_{1}+N, \eta_{2}-\sum_{h=1}^{N} \log \left(1-V_{h}\right)\right)
$$

Note that this Gibbs sampling algorithm consists only of simple steps for sampling from standard distributions and is no more complex than blocked Gibbs samplers 
for DPMs. In addition, we have observed good computational performance, in terms of mixing and convergence rates, in simulated and real data applications.

\section{Simulation examples}

We obtained data from two simulated examples, where $n=500$ and a univariate predictor $x_{i}$ was simulated from Uniform $(0,1)$. Case 1 was a null case where $y_{i}$ was generated from a normal regression model $N\left(y_{i} ;-1+2 x_{i}, 0.01\right)$. Case 2 was a mixture of two normal linear regression models, with the mixture weights depending on the predictor, with the error variance differing, and with a nonlinear mean function for the second component:

$$
f\left(y_{i} \mid \mathbf{x}_{i}\right)=e^{-2 x_{i}} N\left(y_{i} ; x_{i}, 0.01\right)+\left(1-e^{-2 x_{i}}\right) N\left(y_{i} ; x_{i}^{4}, 0.04\right) .
$$

We applied the $\mathrm{IDPM}^{N}$ model in (12) to the simulated data with $N=50$. Based on the results, $N=50$ seems to be chosen to be large enough since the higher clusters having higher indices are not used in any of the subjects or are used in only a small proportion of them.

Also, repeating the analysis for twice $N$, we obtained very similar results, suggesting that the results are robust to the choice of $N$, as long as $N$ is not chosen to be small.

For the hyperparameters, we let $\nu_{1}=\nu_{2}=0.01, \eta_{1}=\eta_{2}=2, \nu_{0}=p, \Sigma_{0}=\boldsymbol{I}_{p}, \boldsymbol{\mu}_{0}=\mathbf{0}, \Sigma_{\mu}=$ $n\left(\mathbf{X}^{\prime} \mathbf{X}\right)^{-1}, a_{\Gamma}=-0.05$, and $b_{\Gamma}=1.05$. The neighborhood size $\psi=0.05$ was chosen such that the average number of subjects belonging to the neighborhoods around each predictor value in the sample is $\approx n / 10$. We analyzed the simulated data using the proposed Gibbs sampling algorithm run for 10,000 iterations with a 5,000 iteration burn-in. The convergence and mixing of the MCMC algorithm were good (trace plots not shown). Also, results tended to be robust to repeating analysis with reasonable alternative hyperparameter values.

For Case 1, as shown in Fig. 3, the predictive mean regression curve (blue dashed, right bottom panel), the true linear regression function (red solid), and the pointwise $95 \%$ credible intervals (green dashed) were almost the same. Figure 3 also shows the predictive densities (blue dashed) at the 10th, 25th, 50th, 75th, and 90th sample percentiles of $x_{i}$, with these densities almost indistinguishable from the true densities (red solid).

For Case 2, Fig. 4 shows an $x-y$ plot (right bottom panel) of the data along with the estimated predictive mean curve (blue dashed), which closely follows the true mean curve (red solid). Figure 4 also shows the estimated predictive densities (blue dashed) correspond approximately to the true densities (red solid) in most cases and the $95 \%$ credible intervals (green dashed) closely cover the true densities in all cases.

Repeating the analysis for Case 2, but with $\beta_{i} \stackrel{\text { iid }}{\sim} G$ and $G \sim \operatorname{DP}\left(a G_{0}\right)$, we obtained poor results estimates diverged substantially from true densities, posterior mean curve failed to capture true nonlinear function), suggesting that a DPM model is inadequate.

\section{Epidemiological application}

\subsection{Background and motivation}

In diabetic studies, interest often focuses on the relationship between 2-h serum insulin levels (indicator for insulin sensitivity/resistence) and 2-h plasma glucose levels (indicator for diabetic risk) that are measured in the oral glucose tolerance test (OGTT). Although most studies examine the mean change of the 2-h insulin versus 2 -h glucose, it would be more interesting to assess the whole distributional change of the 2-h insulin level across the range of the 2-h glucose levels. 
We obtained data from a study which followed a sample of Pima Indians from a population near Phoenix, Arizona since 1965. This study was conducted by the National Institute of Diabetes and Digestive and Kidney Disease, with the Pima Indians chosen because of their high risk of diabetes. Using these data, our goal is conducting inferences on changes in the 2-h serum insulin distribution with changes in 2-h glucose level without making restrictive assumptions, such as normality or a constant residual variation. Certainly, it is biologically plausible that the insulin distribution is non-normal and should change as the glucose level changes not only in mean but also in other features such as skewness, residual variation, and modality.

\subsection{Analysis and results}

For woman $i(i=1, \ldots, 393)$, let $y_{i}$ correspond to the 2 -h serum insulin level measured in $\mu \mathrm{U} / \mathrm{ml}$ (micro Units per milliliter) and let $x_{i}$ denote the 2-h plasma glucose level measured in $\mathrm{mg} / \mathrm{dl}$ (milligrams per deciliter). We applied the $\mathrm{IDPM}^{N}$ model described in (12), after scaling $y$ and $x$ by dividing by 100 . Hyperparameters were set to be the same as in the simulation study except that $\psi=0.08$ such that $n / 10$ subjects belong to each neighborhood on average and $a_{\Gamma}=\min \left(x_{i}\right)-\psi$, and $b_{\Gamma}=\max \left(x_{i}\right)+\psi$ ) such that the edge effects are avoided in the inference. We analyzed the data using the proposed Gibbs sampling algorithm run for 10,000 iterations with a 5,000 iteration burn-in. The convergence and mixing of the MCMC algorithm were good (Trace plots not shown) and results were robust with reasonable alternative hyperparameter values.

Figure 5 shows the predictive distributions for the insulin level at various empirical percentiles of the glucose level. As the glucose level increases, there is a slightly nonlinear change in the mean insulin level (right bottom panel) and a dramatic increase in the heaviness of the right tail of the insulin distribution. Also, some multi-modality in the insulin distribution appears as the glucose level falls into the pre-diabetes range (140$200 \mathrm{mg} / \mathrm{dl})$ and closer to the cut point $(200 \mathrm{mg} / \mathrm{dl})$ for the diagnosis of diabetes. This shift in the shape of the insulin distribution biologically implies that the women with pre-diabetes are expected to have different insulin sensitivities, which may further induce different diabetic risks even for the same glucose level. This may be due to unadjusted covariates or unmeasured risk factors. Such distributional changes in response induced by predictors (e.g. risk factor, exposure, treatment, and, etc.) is pervasive in epidemiologic studies, but is not at all well characterized by standard regression models that do not allow the whole distribution to flexibly change with predictors.

\section{Discussion}

This article proposed a new SBP for the collection of predictor-dependent random probability measures. The prior, called the IDP, is a useful alternative to recently developed prior models that induce predictor-dependence among distributions. Its marginal DP structure should be useful in considering theoretical properties, such as posterior consistency and rates of convergence. A related formulation was independently developed by Griffin and Steel (2008) although the IDP is appealing in its simplicity for construction and computation. In particular, the construction is intuitive and leads to simple expressions for the dependence in random measures at different locations, while also leading to straightforward posterior computation relying on truncation with a fair amount of accuracy.

Although we have focused on a conditional density estimation application, there are many interesting applications of the IDP to be considered in future work. First, the DP is widely used to induce a prior on a random partition or clustering structure (Quintana 2006; Kim et al. 2006). In such settings, the DP has the potential disadvantage of requiring an exchangeability assumption, which may be violated when predictors are available that can 
inform about the clustering. The IDP provides a straightforward mechanism for local, predictor-dependent clustering, which can be used as an alternative to product partition models (Quintana and Iglesias 2003) and model-based clustering approaches (Fraley and Raftery 2002). It is of interest to explore the theoretical properties of the induced prior on the random partition. In this respect, it is likely that the hyperparameter $\psi$ plays a key role. Hence, as a more robust data-driven approach one may consider fully Bayes or empirical Bayes methods for allowing uncertainty in $\psi$.

\section{Acknowledgments}

This research was supported in part by the Intramural Research Program of the NIH, National Institute of Environmental Health Sciences.

\section{Appendix}

Proof of Lemma 1 An infinite number of locations $\Gamma=\left\{\Gamma_{h}, h=1, \ldots, \infty\right\}$ are generated from $H$ on $\mathscr{X}^{\prime}$. Any $\psi$-neighborhood of $\mathbf{x}$ defined as $\eta_{\mathbf{x}}^{\psi}=\left\{\mathbf{x}^{\prime}: d\left(\mathbf{x}, \mathbf{x}^{\prime}\right)<\psi, \mathbf{x}^{\prime} \in \mathscr{X}^{\prime}\right.$ with $\psi>0$ is a subsect of $\mathscr{X}^{\prime}$. The regularity Condition 1 for $H$ ensures that there is a positive probability for a location $\Gamma_{h}$ to be generated in any $\eta_{\mathbf{x}}^{\psi}$. Therefore, there are also an infinite number of locations in $\eta_{\mathbf{x}}^{\psi}$ for all $\mathbf{x} \in \mathscr{X}$ and $\psi>0$, which implies $N(\mathbf{x})=\infty$. Then, $\sum_{l=1}^{N(\mathbf{x})} p_{l}(\mathbf{x})$ almost surely by Lemma 1 in Ishwaran and James (2001).

Proof of Theorem 1 Assume that $\mathscr{G}_{\mathscr{X}} \operatorname{IDP}\left(\alpha, G_{0}, H, \psi\right)$. Then, from the definition of the IDP in (5)-(7), we can reexpress (7) as $G_{\mathbf{x}}=\sum_{l=1}^{N(\mathbf{x})} V_{l}^{(\mathbf{x})} \prod_{j<l}\left(1-V_{j}^{(\mathbf{x})}\right) \delta_{\theta_{l}^{(\mathbf{x})}}$, where $V_{l}^{(\mathbf{x})}$ is the $t$ th element of $\mathbf{V}(\mathbf{x})$ and $\theta_{l}^{(\mathbf{x})}$ is the th element of $\Theta(\mathbf{x})$. Note that it follows from the proof of Lemma 1 that $N(\mathbf{x})=\infty$. Since the random weights and atoms are generated by iid sampling from $\operatorname{Beta}(1, a)$ and $G_{0}$, respectively, independently from the location, we have $V_{l}^{(\mathbf{x})} \stackrel{\underset{\sim}{\mathrm{iid}}}{\operatorname{Beta}}(1, \alpha)$ independently from $\Theta_{l}^{(\mathbf{x})} \stackrel{\underset{\sim}{\mathrm{iid}}}{\sim} G_{0}$, for $l=1, \ldots, \infty$. Hence, it follows directly from Sethuraman's (1994) representation of the DP, that $G_{\mathbf{x}} \mathrm{DP}\left(\alpha G_{0}\right), \forall_{\mathbf{x}} \in \mathscr{X}$.

Proof of Theorem 2 Given $\Gamma$ and $\mathbf{V}$,

$$
\begin{aligned}
\operatorname{Pr}\left(\phi_{i}=\phi_{j} \mid \mathbf{x}_{i}, \mathbf{x}_{j}, \Gamma, \mathbf{V}, \psi\right) & =\sum_{\left\{(k, l): \pi_{k}\left(\mathbf{x}_{i}\right)=\pi_{l}\left(\mathbf{x}_{j}\right)\right\}} p_{k}\left(\mathbf{x}_{i}\right) p_{l}\left(\mathbf{x}_{j}\right) \\
& =\sum_{h \in \mathscr{L}_{\mathbf{x}_{j}} \cap \mathscr{L}_{\mathbf{x}_{j}}} V_{h}^{2} \prod_{m \in \mathscr{S}_{h}}^{2}\left(1-V_{m}\right)^{2} \prod_{n \in \mathscr{S}_{h}^{\prime}}\left(1-V_{n}\right) .
\end{aligned}
$$

For the definition of $\mathscr{S}_{h}$ and $\mathscr{S}_{h}^{\prime}$, refer to the Eq. (9) in Sect. 3.2. Marginalizing out $\mathbf{V}$ over the Beta distribution,

$$
\operatorname{Pr}\left(\phi_{i}=\phi_{j} \mid \mathbf{x}_{i}, \mathbf{x}_{j}, \Gamma, \alpha, \psi\right)=\frac{2}{(\alpha+1)(\alpha+2)} \sum_{h \in \mathscr{L}_{\mathbf{x}_{i}} \cap \mathscr{L}_{\mathbf{x}_{j}}}\left(\frac{\alpha}{\alpha+2}\right)^{\# \mathscr{S}_{h}}\left(\frac{\alpha}{\alpha+1}\right)^{\# \mathscr{S}_{h}^{\prime}}
$$

In order to marginalize out $\mathscr{S}_{h}$ and $\mathscr{S}_{h}^{\prime}$, we introduce $Z_{\gamma_{j}} \stackrel{\text { iid }}{\sim}$ Bernoulli $\left(P_{\mathbf{x}_{i}, \mathbf{x}_{j}}\right)$ as described in the formulations from (9) to (10) in Sect. 3.2. Then, 


$$
\operatorname{Pr}\left(\phi_{i}=\phi_{j} \mid \mathbf{x}_{i}, \mathbf{x}_{j}, \Gamma, \alpha, \psi\right)=\frac{2}{(\alpha+1)(\alpha+2)} \sum_{j=1}^{\infty} \mathrm{Z}_{\gamma_{j}}\left(\frac{\alpha}{\alpha+2}\right)^{\sum_{k=1}^{j-1} \mathrm{Z}_{\gamma_{k}}} \times\left(\frac{\alpha}{\alpha+1}\right)^{j-1-\sum_{k=1}^{j-1} \mathrm{Z}_{\gamma_{k}}} .
$$

(After marginalizing out the $\left\{Z_{\gamma_{j}}\right\}_{j=1}^{\infty}$ as in the Proof of Theorem 3, we obtain:

$$
\begin{aligned}
\operatorname{Pr}\left(\phi_{i}=\phi_{j} \mid \mathbf{x}_{i}, \mathbf{x}_{j}, \alpha, \psi\right) & =\left[\frac{2}{(\alpha+1)(\alpha+2)}\right]\left[\frac{P_{\mathbf{x}_{i}, \mathbf{x}_{j}}(\alpha+2)(\alpha+1)}{\alpha\left(1+P_{\mathbf{x}_{i}, \mathbf{x}_{j}}\right)+2}\right] \\
& =\frac{2 P_{\mathbf{x}_{i}, \mathbf{x}_{j}}}{\left(1+P_{\mathbf{x}_{i}, \mathbf{x}_{j}}\right) \alpha+2} .
\end{aligned}
$$

Proof of Theorem 3 From (10),

$$
\operatorname{Corr}\left\{G_{\mathbf{x}_{i}}(B), G_{\mathbf{x}_{2}}(B) \mid \Gamma\right\}=\frac{2}{\alpha+2} \sum_{j=1}^{\infty} \mathrm{Z}_{\gamma_{j}}\left(\frac{\alpha+1}{\alpha+2}\right)^{j=1} \sum_{k=1}^{j-1} \mathbf{Z}_{\gamma_{k}}\left(\frac{\alpha}{\alpha+1}\right)^{j-1},
$$

where $Z_{\gamma j}$ are iid draws from Bernoulli $\left(P_{\mathbf{x}_{1}, \mathbf{x}_{2}}\right)$. Taking expectation of $\left\{Z_{\gamma_{j}}\right\}_{j=1}^{\infty}$ with respect to Bernoulli $\left(\left(P_{\mathbf{x}_{1}, \mathbf{x}_{2}}\right)\right)$,

$$
E\left[\operatorname{Corr}\left\{G_{\mathbf{x}_{i}}(B), G_{\mathbf{x}_{2}}(B)\right\}\right]=\frac{2}{\alpha+2} P_{\mathbf{x}_{1}, \mathbf{x}_{2}} \sum_{j=1}^{\infty}\left(\frac{\alpha}{\alpha+1}\right)^{j-1} E\left[\left(\frac{\alpha+1}{\alpha+2}\right)^{Y_{j}}\right],
$$

where $Y_{j} \sim \operatorname{Binomial}\left(j-1, P_{\mathbf{x}_{1}, \mathbf{x}_{2}}\right)$. Using the Binomial Theorem, the expectation on the right is marginalized out with respect to $\operatorname{Binomial}\left(j-1, P_{\mathbf{x}_{1}, \mathbf{x}_{2}}\right)$, which results in

$$
\operatorname{Corr}\left\{G_{\mathbf{x} 1}(B), G_{\mathbf{x} 2}(B)\right\}=\frac{2}{\alpha+2} P_{\mathbf{x}_{1}, \mathbf{x}_{2}} \sum_{j=1}^{\infty}\left[\frac{-\alpha P_{\mathbf{x}_{1}, \mathbf{x}_{2}}}{(\alpha+2)(\alpha+1)}+\frac{\alpha}{\alpha+1}\right]^{j-1} .
$$

Since $\left|\frac{-\alpha P_{\mathbf{x}_{1}, \mathbf{x}_{2}}}{(\alpha+2)(\alpha+1)}+\frac{\alpha}{\alpha+1}\right| \leq 1$, the infinite sum on the right converges. Then,

$$
\operatorname{Corr}\left\{G_{\mathbf{x}_{1}}(B), G_{\mathbf{x}_{2}}(B)\right\}=\left(\frac{2 P_{\mathbf{x}_{1}, \mathbf{x}_{2}}}{\alpha+2}\right)\left(\frac{(\alpha+2)(\alpha+1)}{\alpha\left(1+P_{\mathbf{x}_{1}, \mathbf{x}_{2}}\right)+2}\right)=\frac{2 P_{\mathbf{x}_{1}, \mathbf{x}_{2}}(\alpha+1)}{\left(1+P_{\mathbf{x}_{1}, \mathbf{x}_{2}}\right) \alpha+2} .
$$

Proof of Theorem 4 Due to the marginal DP property and using the inequality on the left in (11) with $n=1$, we get $\left\|\mu_{N}(\mathbf{x})-\mu_{\infty}(\mathbf{x})\right\| \leq 4\left(1-E\left[\left(\sum_{h=1}^{N(\mathbf{x})-1} p_{h}\right)\right]\right)$, where $\mu_{N}, \mu_{\infty}, N$ in (11) are replaced by $\mu_{N}(\mathbf{x}), \mu_{\infty}(\mathbf{x}), N(\mathbf{x})$, respectively, and $n$ is substituted by 1 . Here, $N(\mathbf{x})$ is random differently from $N$ in (11). Conditioned on $N(\mathbf{x})$ but marginalizing out $p_{h}$, we get $\left\|\mu_{N}(\mathbf{x})-\mu_{\infty}(\mathbf{x})\right\| \leq 4 E\left[\left(\frac{\alpha}{1+\alpha}\right)^{N(\mathbf{x})-1}\right]$. Note that $N(\mathbf{x}) \sim \operatorname{Binomial}\left(N, P_{\mathbf{x}}\right)$ as discussed in Sect. 
3.3. Then, using the Binomial Theorem, we obtain

$$
\left\|\mu_{N}(\mathbf{x})-\mu_{\infty}(\mathbf{x})\right\| \leq 4\left(\frac{\alpha+1}{\alpha}\right)\left[1-\left(\frac{1}{\alpha+1}\right) P_{\mathbf{x}}\right]^{N}
$$

\section{References}

Beal, M.; Ghahramani, Z.; Rasmussen, C. Neural information processing systems. Vol. 14. MIT Press; Cambridge: 2002. The infinite hidden Markov model.

Blei, D.; Griffiths, T.; Jordan, M.; Tenenbaum, J. Neural information processing systems. Vol. 16. MIT Press; Cambridge: 2004. Hierarchical topic models and the nested Chinese restaurant process.

Caron, F.; Davy, M.; Doucet, A.; Duflos, E.; Vanheeghe, P. Bayesian inference for dynamic models with Dirichet process mixtures; International conference on information fusion; Italia. 2006; Jul $10-13$.

De Iorio M, Müller P, Rosner GL, MacEachern SN. An Anova model for dependent random measures. Journal of the American Statistical Association. 2004; 99:205-215.

Dowse KG, Zimmet PZ, Alberti GMM, Bringham L, Carlin JB, Tuomlehto J, Knight LT, Gareeboo H. Serum insulin distributions and reproducibility of the relationship between 2- hour insulin and plasma glucose levels in Asian Indian, Creole, and Chinese Mauritians. Metabolism. 1993; 42:1232-1241. [PubMed: 8412735]

Duan, JA.; Guidani, M.; Gelfand, AE. ISDS Discussion Paper, 05-23. Duke University; Durham: 2005. Generalized spatial Dirichlet process models.

Dunson DB. Bayesian dynamic modeling of latent trait distributions. Biostatistics. 2006; 7:551-568. [PubMed: 16488893]

Dunson DB, Park J-H. Kernel stick-breaking process. Biometrika. 2008; 95:307-323. [PubMed: 18800173]

Dunson DB, Peddada SD. Bayesian nonparametric inference on stochastic ordering. Biometrika. 2008; 95:859-874.

Dunson DB, Pillai N, Park J-H. Bayesian density regression. Journal of the Royal Statistical Society, Series B. 2007; 69:163-183.

Escobar MD. Estimating normal means with a Dirichlet process prior. Journal of the American Statistical Association. 1994; 89:268-277.

Escobar MD, West M. Bayesian density estimation and inference using mixtures. Journal of the American Statistical Association. 1995; 90:577-588.

Ferguson TS. A Bayesian analysis of some nonparametric problems. The Annals of Statistics. 1973; 1:209-230.

Ferguson TS. Prior distributions on spaces of probability measures. The Annals of Statistics. 1974; 2:615-629.

Fraley C, Raftery AE. Model-based clustering, discriminant analysis, and density estimation. Journal of the American Statistical Association. 2002; 97:611-631.

Gelfand AE, Kottas A, MacEachern SN. Bayesian nonparametric spatial modeling with Dirichlet process mixing. Journal of the American Statistical Association. 2004; 100:1021-1035.

Ghosal S, Van der Vaart AW. Posterior convergence rates of Dirichlet mixtures at smooth densities. The Annals of Statistics. 2007; 35(2):697-723.

Ghosal S, Ghosh JK, Ramamoorthi RV. Posterior consistency of Dirichlet mixtures in density estimation. The Annals of Statistics. 1999; 27:143-158.

Griffin JE, Steel MFJ. Order-based dependent Dirichlet processes. Journal of the American Statistical Association. 2006; 101:179-194.

Griffin, JE.; Steel, MFJ. Bayesian nonparametric modeling with the Dirichlet process regression smoother. Technical Report. University of Warwick; 2008.

Ishwaran H, James LF. Gibbs sampling methods for stick-breaking priors. Journal of the American Statistical Association. 2001; 96:161-173.

Kim S, Tadesse MG, Vannucci M. Variable selection in clustering via Dirichlet process mixture models. Biometrika. 2006; 94:877-893. 
Lijoi A, Prünster I, Walker SG. On consistency of non-parametric normal mixtures for Bayesian density estimation. Journal of the American Statistical Association. 2005; 100:1292-1296.

Lo AY. On a class of Bayesian nonparametric estimates: I. Density estimates. The Annals of Statistics. 1984; 12:351-357.

MacEachern, SN. Dependent nonparametric processes; ASA proceedings of the section on bayesian statistical science; Alexandria. 1999; American Statistical Association;

MacEachern, SN. Dependent Dirichlet processes. Department of Statistics, The Ohio State University; 2000. Unpublished manuscript

MacEachern, SN. Decision theoretic aspects of dependent nonparametric processes. In: George, E., editor. Bayesian methods with applications to science, policy and official statistics. ISBA; Creta: 2001. p. 551-560.

Müller P, Quintana F, Rosner G. A method for combining inference across related nonparametricBayesian models. Journal of the Royal Statistical Society B. 2004; 66:735-749.

Pennell ML, Dunson DB. Bayesian semiparametric dynamic frailty models for multiple event time data. Biometrics. 2006; 62:1044-1052. [PubMed: 17156278]

Pitman J. Exchangeable and partially exchangeable random partitions. Probability Theory and Related Fields. 1995; 102:145-158.

Pitman, J. Some developments of the Blackwell-MacQueen urn scheme. In: Ferguson, TS.; Shapley, LS.; MacQueen, JB., editors. Statistics, probability and game theory. Vol. 30. Institute of Mathematical Statistics; Hayward: 1996. p. 245-267.IMS Lecture Notes-Monograph Series

Quintana FA. A predictive view of Bayesian clustering. Journal of Statistical Planning and Inference. 2006; 136:2407-2429.

Quintana FA, Iglesias PL. Bayesian Clustering and product partition models. Journal of the Royal Statistical Society B. 2003; 65:557-574.

Sethuraman J. A constructive definition of the Dirichlet process prior. Statistica Sinica. 1994; 2:639_ 650.

Smith, JW.; Everhart, JE.; Dickson, WC.; Knowler, WC.; Johannes, RS. Using the ADAP learning algorithm to forecast the onset of diabetes mellitus; Proceedings of the symposium on computer applications in medical care; 1988; p. 261-265.

Xing, EP.; Sharan, R.; Jordan, M. Bayesian haplotype inference via the Dirichlet process; Proceedings of the international conference on machine learning (ICML); 2004. 


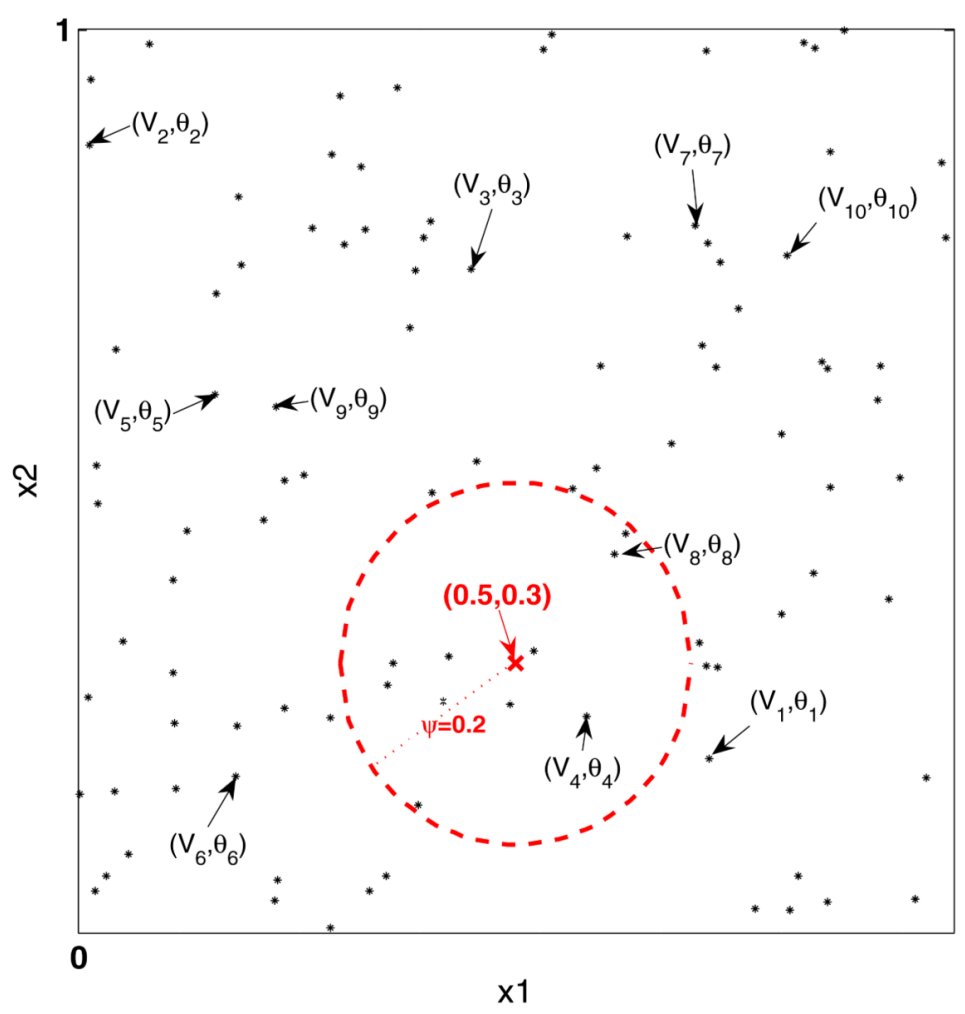

Fig. 1.

Graphical illustration for 1DP formulation. Black asterisks are the first 100 random locations generated on $\mathscr{X}^{\prime}=[0,1]^{2}$ from $H=$ Uniform $\left([0,1]^{2}\right)$. Red dashed circle indicates the neighborhood of the red crossed predictor point $\mathbf{x}=(0.5,0.3)^{\prime}$ determined by Euclidian distance $\mathrm{d}(\cdot, \cdot)$ and $\psi=0.2$. $\left(V_{h}, \theta_{h}\right)$ for $h=1, \ldots, 10$ are the first ten random pairs of weight and atom assigned to the first ten random locations $\Gamma_{h}$ for $h=1, \ldots, 10$ 

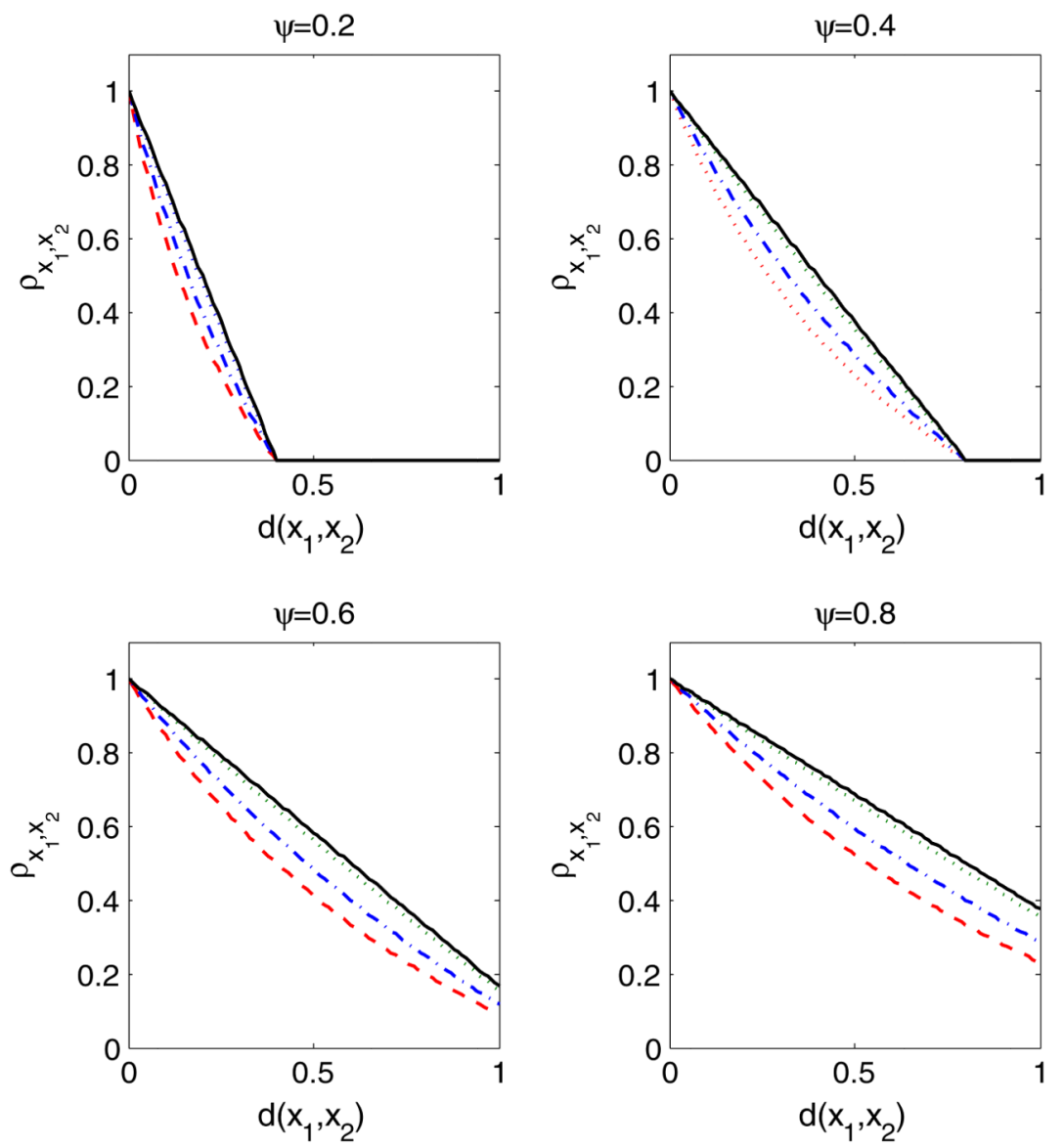

Fig. 2.

Change in correlation $\rho_{\mathbf{x}_{1}, \mathbf{x}_{2}}$ over the change in distance $d\left(\mathbf{x}_{1}, \mathbf{x}_{2}\right)$ for different $a$ and $\psi: a=$ 0.0001 (red dashed), $a=1$ (blue dot-dashed), $a=10$ (green dotted), $a=10,000$ (black solid) 

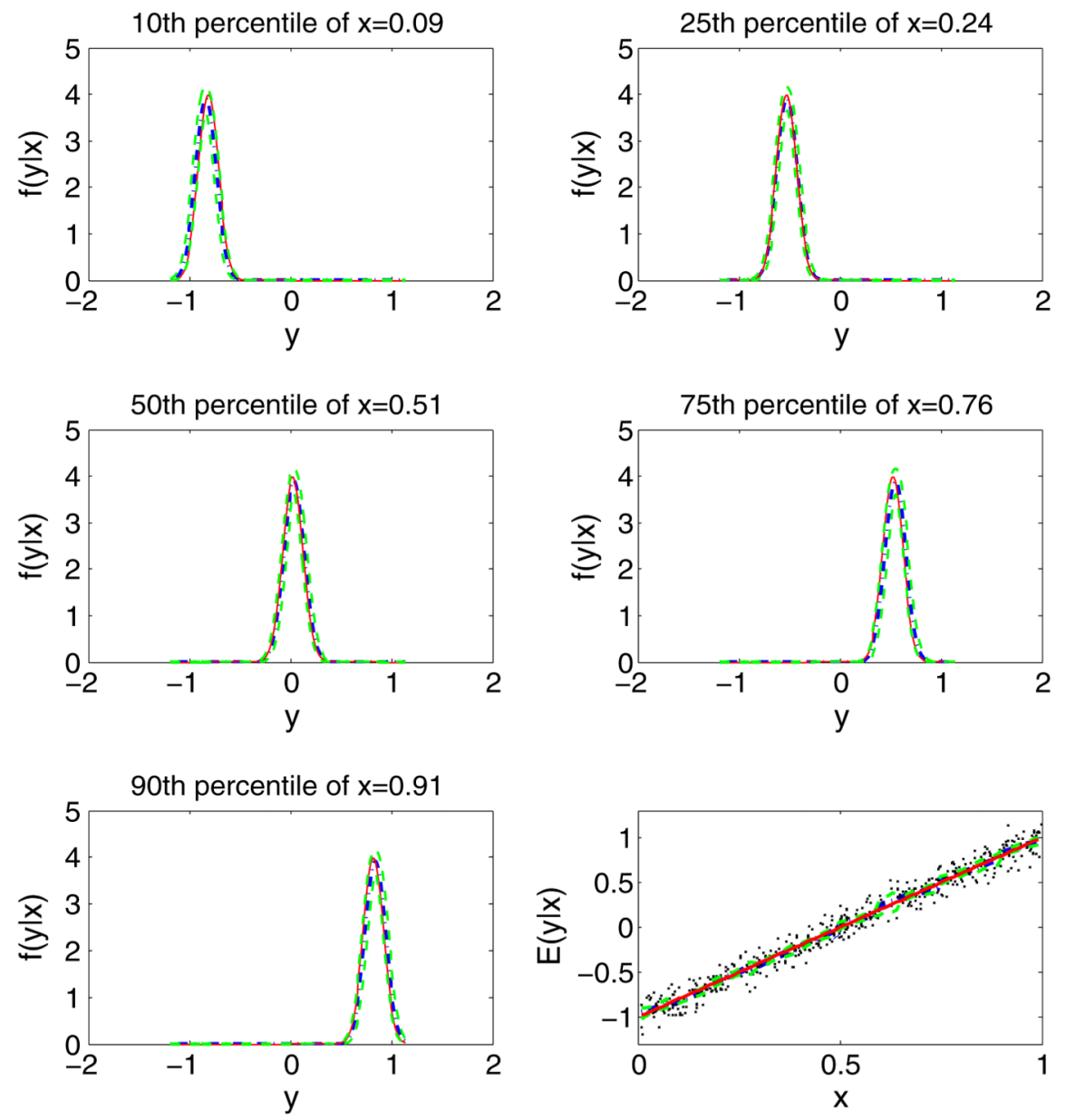

Fig. 3.

Results for simulation Case 1: true conditional densities of $y \mid x$ (red solid), predictive conditional densities (blue dot-dashed), and $95 \%$ pointwise credible intervals (green dashed). The lower right panel shows the data (black dots), along with true (red solid) and estimated mean (blue dashed) regression curves superimposed with $95 \%$ credible line (green dashed) 

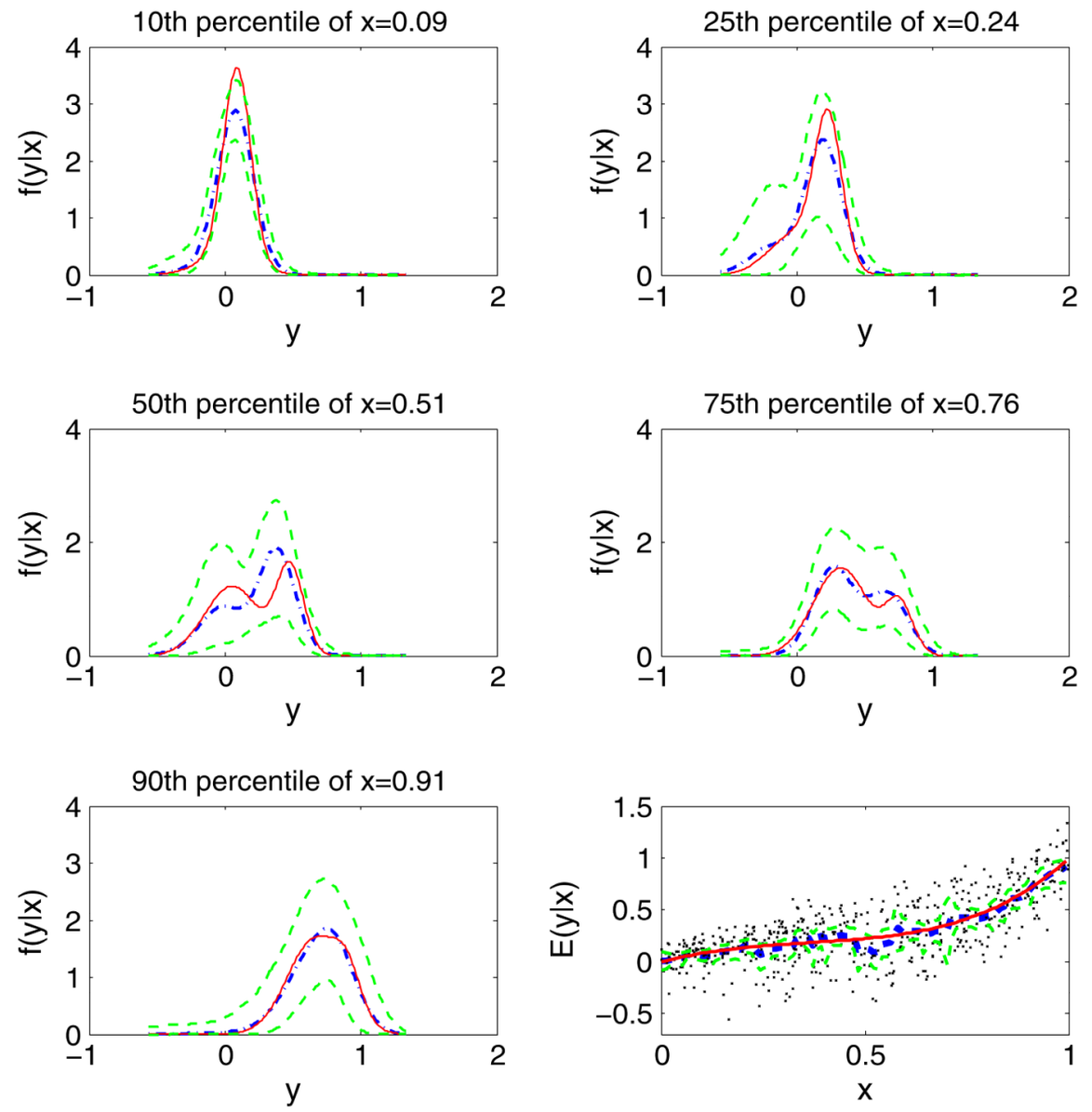

Fig. 4.

Results for simulation Case 2: true conditional densities of $y \mid x$ (red solid), predictive conditional densities (blue dot-dashed), and $95 \%$ pointwise credible intervals (green dashed). The lower right panel shows the data (black dots), along with true (red solid) and estimated mean (blue dashed) regression curves superimposed with $95 \%$ credible line (green dashed) 

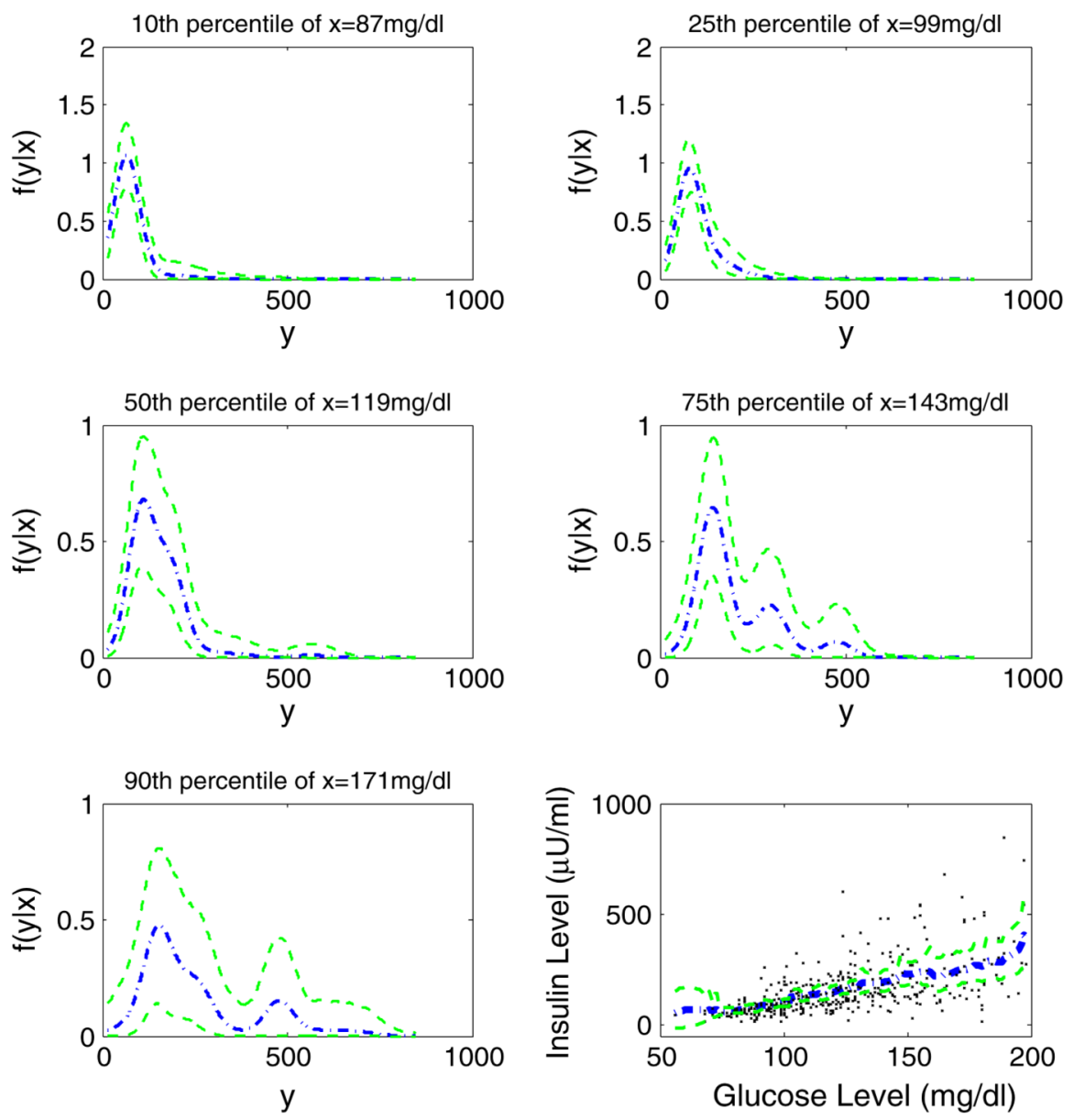

Fig. 5.

Results for Pima Indian Example: predictive conditional densities (blue dot-dashed), and 95\% pointwise credible intervals (green dashed). The lower right panel shows the data (black dots), along with estimated mean (blue dashed) regression curves superimposed with 95\% credible line (green dashed) 